\title{
ESSAI DE CARACTERISATION DU TWEET POLITIQUE
}

Le propos de cet article est de procéder à une analyse de tweets émis par des personnalités politiques, ou des partis politiques, afin de tenter de cerner quelles sont les caractéristiques d'une forme récente de communication, qui fait coïncider, dans un même message, un certain nombre de contraintes spécifiques. En effet, il y a originellement dans Twitter un caractère spontané des productions envoyées sur le réseau, mais cette spontanéité n'est pas forcément maintenue dans le cadre du discours politique ${ }^{1}$. Le support et le discours pèsent donc sur les écrits, et l'analyse devrait permettre de mesurer de quelle manière chacun agit sur les productions. Un tweet a également une taille maximum (140 caractère), ce qui conduit à un certain nombre d'ajustements habituellement absents du discours politique. Enfin, Twitter étant un outil numérique, sa dimension technolangagière (Paveau 2012) modifie à la fois l'aspect pragmatique des messages qui sont transmis, mais également la grammaire des formes langagières. Aussi, si des considérations traditionnellement convoquées en pragmatique ou analyse du discours sont présentes dans les tweets que nous analyserons (ethos, caractère formulaire, etc.), d'autres dimensions permettront de concevoir le tweet non comme un support ou canal supplémentaire de diffusion de l'information, mais comme une forme d'expression. Aussi, le tweet politique pourrait s'apparenter, comme nous essaierons de le montrer, à un genre de discours à part entière, qui permet la constitution d'un ethos discursif, accompagné de composants technologiques du réseau qui aboutiraient finalement à la constitution d'un ethos technodiscursif, voire prétechnodiscursif dans certains cas.

\section{LE RESEAU TWITTER ET L'USAGE FAIT EN POLITIQUE : UN GENRE DE DISCOURS}

Avant de procéder à des analyses de détail de tweets politique, présentons rapidement Twitter :

Twitter est un service de microblogage, permettant aux utilisateurs de bloguer grâce à des messages courts (140 caractères maximum, soit une ou deux phrases). Outre cette concision imposée, la principale différence entre Twitter et un blog traditionnel réside dans le fait que Twitter n'invite pas les lecteurs à commenter les messages postés. Le slogan d'origine de Twitter, What are you doing? le définissait comme un service permettant de raconter ce qu'on fait au moment où on le fait. Prenant acte de l'utilisation du service pour s'échanger des informations et des liens, Twitter le remplace par What's happening? («Quoi de neuf? » ou encore « Que se passe-t-il ? » dans la version française).

Ce réseau permet d'exprimer habituellement des éléments d'actualité de manière brève et concise. Il permet également de «tweeter» des liens vers des sites que l'auteur du tweet recommande (souvent un billet sur un blog, un article de presse). Une grande majorité des personnalités politiques ont actuellement un compte Twitter, et les partis politiques ont également un compte qui rend compte des informations du parti et de ses membres.

Il y a un intérêt à travailler les tweets du point de vue des sciences du langage, tel que cela est notamment explicité par Paveau (2012: en ligne) qui recommande de «travailler les productions technolangagières et technodiscursives et par conséquent d'observer des configurations technolangagières ou - discursives c'est-à-dire des productions discursives

\footnotetext{
${ }^{1}$ Nous envisageons ici le discours au sens de Benveniste, comme «énonciation supposant un locuteur et un auditeur, et chez le premier l'intention d'influencer l'autre » (1966:242), et ainsi comme acte de communication déterminé par des conditions socio-historiques, dont rendent compte les genres de discours. L'hypothèse que nous examinons ici consiste à appréhender les «énonciations » produites par tweets, en les considérant du point de vue des spécificités des conditions socio-historiques (donc appréhender le tweet politique comme un genre du discours politique, au même titre que l'allocution, le communiqué, etc.), même si certaines nuances méthodologiques seront apportées par la suite.
} 
dans leur environnement et leur nature technologique ». Il y a en effet, selon elle, des «traits technodicursifs du genre ou de l'écriture (twittécriture) », qui sont listés comme suit :

La fameuse contrainte des 140 signes : l'usage sur Twitter est de ne pas abréger, de ne pas adopter le langage sms (ceux qui le pratiquent sont repris assez couramment), contrainte cependant contournable par le raccourcissement des liens, les tweets en plusieurs tweets $(1 / 3,2 / 3$, etc.).

Les contraintes/transgressions grammaticales.

Des aménagements typographiques : espaces, ponctuation.

Mais surtout la délinéarisation de l'énoncé : par insertion de liens, de hashtags, d'énonciateurs/interlocuteurs multiples, les trois étant cliquables; il s'agit donc d'une double délinéarisation, syntagmatique et hypertextuelle, visible sur l'écran puisque tout ce qui est cliquable apparaît en couleur.

Méthodologiquement, la recherche a été menée à partir d'événements politiques, pour lesquels nous avons recensé un ensemble de tweets, qui constituent un petit corpus d'étude dont nous rendons compte $\mathrm{ici}^{2}$. Dans ce premier temps de la recherche sur les tweets politiques, l'analyse est qualitative, mais pourrait permettre, dans un second temps, d'aborder ce phénomène d'un point de vue également quantitatif. Les événements en questions sont postérieurs à la campagne présidentielle 2012, et concernent les réactions aux premières actions du gouvernement Ayrault par Marine Le Pen et Jean-Luc Mélenchon, l'expression du Parti Socialiste (désormais PS) et de ses ministres pour justifier leurs actions, et l'affrontement entre Jean-François Copé et François Fillon pour l'accès à la direction de l'Union pour un Mouvement Populaire (désormais UMP).

Sur le plan de la forme des tweets, la taille réduite des productions se prête particulièrement bien à la production de «petites phrases » ou formules, comme c'est le cas dans le discours politique traditionnel, mais ici la singularité d'un tweet comme formule en accentue la force.

\subsection{Les petites phrases sur Twitter : avoir le sens de la formule}

Un exemple en la matière concerne les tweets de Marine Le Pen, via son compte @ MLP_officiel. Si Jean-Marie Le Pen avait l'habitude de commettre des dérapages langagiers, de lancer des petites phrases, qui faisaient parler du Parti et créaient la polémique, Marine Le Pen a rompu partiellement avec le côté polémique de cette stratégie. Cependant, elle n'a pas perdu le sens de la formule, comme dans les exemples ${ }^{3}$ suivants :

http://twitter.com/MLP_officiel/status/217646538971820032

\#Insécurité : aux grands maux, les petits remèdes : http://bit.ly/NwZzEo

$\mathrm{ou}$

http://twitter.com/MLP_officiel/status/227756698717261825

Formation de la « Droite Forte » : l'arnaque de l'été : http://bit.ly/QwNi8J \#DroiteForte \#UMP

Ces tweets s'apparentent selon nous à des «petites phrases », tel que cela est défini dans la synthèse de Krieg-Planque et Olivier-Yaniv (2011 : 18) :

Le terme "petites phrases » sert donc à désigner un ensemble hétérogène de phénomènes. "Petites

\footnotetext{
${ }^{2}$ Il s'agit d'une extraction manuelle, et en cela ce travail n'est pas à proprement parler un travail sur corpus, mais plutôt une étude à partir d'un recueil d'exemples, constitué dans le but de mener une recherche spécifique sur les particularités langagière des tweets politiques. Dans le cadre du groupe «nouv comm » de l'IR corpus, un travail sur l'extraction et l'analyse automatiques de tweets est en cours.

${ }^{3}$ Pour les besoins de l'article, les tweets sont extraits de leur environnement natif et donc présentés hors de leur environnement d'origine. Le lecteur pourra retrouver l'intégralité des tweets cités, dans l'ordre de leur apparition dans l'article, sur la page http://corpusdetweetspolitiques.blogspot.fr/
} 
phrases » est utilisé par de nombreux acteurs sociaux pour décrire des fragments de discours, plus ou moins décontextualisés, qui font l'objet de reprises dans et par les médias, notamment en raison de leur caractère remarquable ou polémique. Pour autant, il se peut que d'autres termes soient utilisés pour qualifier un phénomène apparemment équivalent.

Dans le premier cas, nous avons le pastiche d'une expression figée (Aux grands maux, les grands remèdes), qui est travestie et inversée pour pointer le manque de bon sens (emblématique d'une expression figée) de l'action du gouvernement sur l'insécurité. Dans le second exemple, l'arnaque de l'été transfère la dénomination Droite Forte dans un autre domaine que celui de la politique, puisque l'arnaque de l'été renverrait à des problèmes de consommation et au domaine sociétal. Le jeu sur le langage inauguré par J.-M. Le Pen est donc aussi mis à profit par M. Le Pen, de manière moins polémique, mais plus efficace sur le plan argumentatif. De plus, cette utilisation du potentiel de la langue, des expressions figées, dans des "petites phrases", correspond plutôt bien à l'ancrage nationaliste du parti, qui exploite les ressources de la langue, vue comme trésor dont on peut tirer profit. M. Le Pen use certes de formules qui jouent avec le langage, mais ces formules sont porteuses d'un sens, qui contribue à l'argumentation grâce à la cohérence entre les moyens ludiques et l'idéologie du Parti (avec petits remèdes elle construit une France malade, avec arnaque elle introduit l'idée de la corruption, cohérente avec le « tous pourris » des partis populistes).

Après cette première analyse, remarquons que la méthodologie d'analyse des tweets n'est pas sans poser de problèmes : en effet, la constante évolution du réseau, et les interactions, ne sont pas prises en compte dans la présente étude, ce qui induit une forme de décontextualisation dans les tweets cités. En particulier, un tweet a vocation à être retweeté, il engage un possible échange conversationnel, et son statut plurisémiotique (avec notamment l'avatar qui participe de sa lectorialité) invite à considérer les réactions en série qu'il génère. Pour autant, dans le cadre d'une problématique sur le tweet comme genre du discours politique, le centrement sur la "production» du tweet rend notre analyse comparable à des analyses qui exploreraient d'autres terrains du discours politique (le meeting par exemple génère aussi des réactions, les politiciens réagissent éventuellement à des événements concomitants, etc.). La focalisation sur le tweet politique permet donc selon nous d'en définir malgré tout certaines propriétés (mais pas forcément des productions dans d'autres contextes de la Twittosphère), et d'autres travaux devront être menés par la suite pour compléter la caractérisation du tweet politique par sa dimension interactive.

D'autres tweets jouent sur l'analogie avec le slogan socialiste Le changement, c'est maintenant, permettant à $\mathrm{M}$. Le Pen à la fois d'ironiser sur les promesses du candidat Hollande, et sur les actions en cours :

http://twitter.com/MLP_officiel/status/228489954899152898

Avec \#Valls, les distributeurs automatiques de nationalité, c'est maintenant ! :http://bit.ly/Pv0LMb

ou

http://twitter.com/MLP_officiel/status/220435029308866560

Discours de politique générale : les poncifs c'est maintenant : http://bit.ly/PaOTSe

Des expressions connotées négativement sont substituées au terme changement : distributeurs automatiques de nationalité et les poncifs incarneraient le changement promis par le candidat Hollande, ce qui tourne ses promesses en dérision.

Dans

http://witter.com/MLP_officiel/status/185790680109547520 
"Avec Hollande, le changement c'est comme avant." \#MeetingNice \#MLP2012

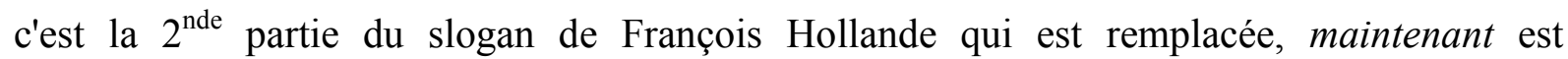
transformé en comme avant. Ceci décrédibilise la notion de changement, centrale dans le programme socialiste, puisque l'aspect de rupture constitutif du mot changement est nié avec comme avant, qui introduit un aspect de continuité. En jouant sur la formule, la candidate tourne non seulement le slogan socialiste en dérision, mais plus profondément elle remet en cause la véracité même de la notion de changement appliquée par les socialistes. Ce trait du tweet politique, présent chez d'autres personnalités politiques, mais particulièrement saillant dans ce cas, s'ajoute à un processus sémantique lié à la brièveté de la formulation, que nous nommons ici la condensation sémantique.

\subsection{La condensation sémantique par décontextualisation partielle ou techno- contextualisation}

En effet, si les tweets peuvent fonctionner comme des «petites phrases », c'est parce qu'ils ont un caractère polémique sur le plan de discours, et parfois excessif sur le plan sémantique, avec le choix de mots qui doivent condenser l'information le plus synthétiquement possible. Ceci permet de créer des discours, ou simplement d'en reprendre, et d'en figer les caractéristiques. Ainsi, par exemple, le 23 septembre, dans Dimanche Plus, Jean-Luc Mélenchon disait à propos du gouvernement : "Un jour, on fait ci, le lendemain on fait ça, le surlendemain n'importe quoi. J'étais mieux préparé à gouverner que cette bande de manchots ». Cette citation n'a pas fait exception à sa reprise en «petite phrase », via le compte Twitter de l'intéressé :

\section{https://twitter.com/JLMelenchon/status/249853784099012608}

"J'étais mieux préparé à gouverner que cette bande de manchots" \#PSA \#DimanchePlus

S'il s'agit de la même « petite phrase », le fil du discours n'est plus présent, et la condensation sémantique (le fait de désigner le gouvernement avec un terme fort de sens) s'appuie sur le fait que le tweet ne véhicule pas avec lui le contexte de production de l'énoncé original : ou s'il le fait, c'est d'une autre manière, par le biais des hashtags, qui véhiculent une forme de contexte, mais de manière stratégique (\#PSA désignant le thème, et \#DimanchePlus le contexte de production) $)^{4}$. La décontextualisation évoquée s'accompagne donc, grâce à Twitter, d'une techno-contextualisation, grâce à l'usage des hashtags, qui importent avec eux des éléments contextuels, et peuvent créer des interactions avec tweets liés aux mêmes contextes. Avec bande de manchots, on interprète l'expression au regard du sens de «pas manchot », qui signifie adroit, habile, et donc «bande de manchots » désigne des personnes malhabiles, en l'occurrence les membres du gouvernement, qu'il juge peu aptes à résoudre les problèmes. Manchots placé comme complément du nom bande indique en outre la dépréciation des individualités qui composent le gouvernement, puisqu'ils sont référencés par un terme flou et péjoratif, doublé d'un complément peu valorisant. Cependant, avec bande, on peut entrevoir pour manchot le sens de l'" oiseau palmipède marin d'assez grande taille, souvent confondu avec le pingouin, auquel les membres antérieurs impropres au vol servent de nageoire » (TLFI). Cet animal ayant une image assez amusante (voir des films ou documentaires sur le sujet, ou les publicités qui utilisent son image), l'ambiguïté est ici productive de sens: cette bande est certes malhabile, mais on peut aussi comparer les individus qui la composent à l'animal amusant, incapable de réussir quelque chose qu'il devrait pouvoir (voler pour le manchot, résoudre une crise avec PSA pour le gouvernement).

\footnotetext{
${ }^{4}$ Ce fonctionnement était aussi à l'œuvre dans les tweets de M Le Pen : c'est pour cela que nous parlons de décontextualisation partielle. Les tweets véhiculent un contexte grâce aux hashtags (voire aux liens, ou aux mentions, seulement ces contextes peuvent déjà servir une forme d'argumentation).
} 
Ceci est d'autant plus possible que le 24 septembre, dans l'émission C'est à vous, J.-L. Mélenchon file cette métaphore animale, reprise dans un tweet :

https://twitter.com/JLMelenchon/status/250284948236431360

"Au gouvernement, ils doivent faire attention de ne pas devenir des pingouins assis sur leur œuf, juste bons à applaudir bêtement" \#Cavous

Avec ne pas devenir des pingouins assis sur leur ouf, juste bons à applaudir bêtement, il mobilise encore un double sens, puisque le pingouin est certes un "oiseau palmipède marin de la famille des Alcidés, au ventre blanc et aux ailes noires, vivant dans l'Atlantique Nord" (TLFI), mais selon le Larousse Encyclopédique "on nomme souvent à tort "pingouins» les manchots, oiseaux spéciaux à l'Antarctique, de constitution très différente, malgré une certaine analogie d'aspect due au plumage noir et blanc et à la station verticale à terre ». Applaudir donne corps à cette interprétation de pingouin, en rapport avec celle de " manchot »-animal, à cause de la petitesse de leurs ailes, qui rend difficile la réalisation de l'applaudissement. Mais dans un registre populaire et péjoratif, le pingouin est aussi "l'individu quelconque, synonyme de bonhomme, type, etc.». De plus, un autre sens de pingouin existe, en rapport avec la façon de s'habiller, comme trouvé sur un forum : «Et faut pas forcement être habillé comme un pingouin (costard cravate) pour être bien habillé ! ». Les sens dans ces deux extraits (avec manchots et pingouins) sont liés au domaine animal ou humain, aux caractéristiques physiques ou aux capacités d'action, et à l'apparence, et convergent toutes vers une caractérisation cohérente de ce que J.-L. Mélenchon attribue au PS : l'incapacité à résoudre les problèmes, le ridicule, le peu de crédit accordé (le gouvernement est une "bande de...»), et un côté endimanché qui contraste avec le socle populaire qu'il représente, en opposition aux riches et au monde de la finance (ceux qui portent le « costard cravate »).

Un autre exemple de cette condensation sémantique grâce au tweet concerne la critique de la classe politique, le rejet des élites et l'appel au peuple par M. Le Pen. Elle revendique en effet l'amalgame entre l'UMP et le PS et l'incarne linguistiquement en popularisant le sigle UMPS :

http://twitter.com/MLP_officiel/status/206371210303512576

Le Président de la République a changé, mais l'UMPS est toujours au pouvoir !http://bit.ly/JkPetd

Certes, ce sigle n'est pas apparu sur Twitter, mais son usage sur le réseau social, dans une forme brève, tend à rendre réelle la reconstruction de l'échiquier. Cet amalgame, et la critique des élites qui l'accompagne, sont décontextualisés d'un fait précis, et sont présentés comme relevant d'une vérité générale. Cette considération s'incarne aussi dans la critique des élites, comme dénomination générique pour désigner la classe politique :

http://twitter.com/MLP_officiel/status/183921043478556672

"Les élites ont pris en France cette très mauvaise habitude de se voiler la face"

Cette vérité proposée est appuyée par l'usage de habitude, et l'expression se voiler la face introduit encore une double lecture, avec un sens «littéral» de l'expression qui renvoie directement à ce qui est dénoncé par $\mathrm{M}$. Le Pen.

\section{DE LA CONSTITUTION D'UN ETHOS DISCURSIF A UN ETHOS TECHNODISCURSIF}

Une des caractéristiques habituellement relevée dans le discours politiques est la constitution de l'ethos de la personnalité politique en situation. Chauvin-Vileno (2002: en ligne) propose une synthèse de l'usage de ce concept en pragmatique et analyse du discours : en référence aux travaux de Amossy et Adam qui montrent que l'ethos a sa place dans la polyphonie énonciative de Ducrot, elle indique que « ce qui est en jeu avec l'ethos, c'est l'importance de 
la représentation de soi et de l'autre dans l'interlocution » Il est utile de distinguer un ethos prédiscursif d'un ethos discursif: l'ethos " repose pour une part sur un savoir préalable des interlocuteurs sur la vie, le caractère, les actions du locuteur, et que ce savoir préalable confère ou non du poids au discours, conditionne la réception ». Ce premier type d'ethos est dit prédiscursif, alors que l'ethos discursif se fonde sur la confiance inspirée par l'orateur par l'effet du discours. L'influence des deux ethos peut se combiner dans les situations concrètes et avoir un degré de pertinence variable selon les types de locuteurs et les genres discursifs considérés. Chauvin-Vileno indique aussi que les images des hommes politiques, créées par la presse, la radio, etc. sont produites et prises dans un circuit discursif (au sens large et non strictement verbal) : «en prenant en compte cet aspect de nos sociétés, l'ethos prédiscursif tendrait à être absorbé par le discursif, ou encore on aurait affaire à des couches successives de discursif ». D. Maingueneau insiste d'ailleurs sur le fait que l'ethos n'est pas attaché au sujet réel mais au sujet dans l'exercice de la parole.

\subsection{L'ethos discursif}

Dans la campagne 2012 pour la conquête de l'UMP, les tweets de Jean-François Copé (@jf_cope) et François Fillon (@FrancoisFillon) rivalisent, chacun essayant de se construire un ethos, certes donc discursif, mais que nous pourrions même appeler technodiscursif. Par exemple, un tweet emblématique de @jf_cope pour se poser en hauteur du débat est celui-ci :

http://twitter.com/jif_cope/status/230637108199555072

Chaque candidature est respectable \& témoigne de la volonté de marquer sa sensibilité, sa diversité \#UMP \#NiceMatinhttp://www.nicematin.com/societe/cope-a-lump-on-ne-doit-pas-avoir-detabou.949781.html ...

Dans ce tweet, que signifie respectable ? A priori, le sens ici est « qui mérite le respect, la considération", en tant que suffixation en -able de "respect», indiquant que les candidats doivent être respectés. Mais dans respectable se dessine aussi un sens «d'une importance quantitative qui mérite d'être prise en considération », comme lorsqu'on dit de quelqu'un qu'il a fait un résultat respectable (généralement correct donc mais insuffisant). @jf_cope s'institue donc au moins comme celui qui est en position de dire que tous les concurrents doivent être respectés (serait-il le favori, dans ce cas ?), ou les crédite d'une certaine valeur qui sera néanmoins insuffisante comparativement à lui.

Avec un fonctionnement assez proche, l'exemple suivant

http://twitter.com/jf_cope/status/230638722796908545

J'ai initié les mouvements qui vont permettre à nos amis de faire connaître leurs différences \#NiceMatinhttp://www.nicematin.com/societe/cope-a-lump-on-ne-doit-pas-avoir-detabou.949781.html ...

incite encore à une double lecture : faire connaître leurs différences ne préfigure aucunement que les amis ont des chance à cette élection, donc les mouvements qui sont censés pouvoir l'emporter dans cette course à l'UMP ne seraient finalement qu'une expression à "faire connaître » et pas un choix qui devrait " convaincre » : en se plaçant du point de vue de l'émission du message (l'information) sans intégrer son impact (la réussite de cette émission), une forme de rabaissement de la parole amie se lit en filigrane, ou plutôt encore une fois la preuve que cette parole est vue et mise en mots par celui qui s'institue favori et en surplomb des débats. Il se constitue donc un ethos discursif de leader de la droite, et se place comme le responsable politique principal.

De son côté, F. Fillon se constitue un ethos discursif plutôt par la manière dont il met en scène sa réalité et son expérience. Dans l'exemple suivant, son engagement politique lui est imposé de l'extérieur, par la gravité de la situation, lui constituant un ethos d'homme providentiel : 
http://es.twitter.com/FrancoisFillon/status/239680595364560896

Apres 5 ans à Matignon j'ai vraiment hésité à continuer la vie politique. Ce qui m'a décidé c'est la gravité de la situation \#Sarthe

et justifie donc le devoir qui est le sien (qu'il lie au rassemblement)

http://es.twitter.com/FrancoisFillon/status/239690894931013632

J'ai un devoir. Tout mettre en œuvre pour le rassemblement Pour l'accomplir j'ai besoin de vous de votre soutien de votre confiance\#Sarthe

et aussi sa solidarité avec les critiques envers N. Sarkozy, afin d'en retirer une légitimité http://es.twitter.com/FrancoisFillon/status/239682474379186176

Pas adepte du droit d'inventaire. Fier du bilan. Toute critique contre NS critique contre moi qui était loyalement à ses côtés pendant 5 ans

avec la mise en exergue de sa loyauté, et de sa proximité avec l'ancien président. L'ancien Premier Ministre se construit donc un ethos discursif d'homme providentiel et d'héritier de l'ancien président. Ce dernier élément est un point fort des tweets des deux protagonistes : se poser comme héritier de l'ancien président. Et c'est F. Fillon qui dans ses discours ou sur Twitter, insiste le plus sur ce point, comme par exemple :

http://twitter.com/FrancoisFillon/status/219190222964015104

Toute critique visant Nicolas Sarkozy me vise en même temps. Je suis solidaire de la totalité de l'action qui a été menée \#Fillon\#UMP \#JDD

Les mots sont précis et bien choisis : solidaire indique l'étroite dépendance introduite entre les deux hommes, et avec critique F. Fillon construit l'image de deux hommes face à l'adversité, qui sont atteints de la même manière, et de façon dépendante. Il évoque aussi leurs relations :

http://twitter.com/FrancoisFillon/status/234207625007476737

Nicolas Sarkozy. De très bonnes relations avec lui. On se parle souvent. Prendre position n'est pas du tout son intention\#UMP \#ouestfrance

Elles sont qualifiées de très bonnes : il tente probablement de montrer qu'il est en meilleure posture que J.-F. Copé de ce point de vue. En effet, ce dernier a parfois eu des relations houleuses avec N. Sarkozy, et il les présente conjointement à son engagement envers l'ancien président. J.-F. Copé tente d'inscrire cet héritage dans la dynamique de ses rapports avec l'ancien président, pour s'adjoindre l'autorité représentée: il qualifie leur relation de singulière, tout en se justifiant de son action :

http://twitter.com/jif_cope/status/220923615674249217

J'ai une relation singulière avec Nicolas Sarkozy, et je me suis pleinement engagé dans sa campagne \#elysee2012

L'usage de singulière est étonnant, dans ce contexte l'adjectif signifie « qui se distingue des autres, qui est unique en son genre » (voir le TLFI), et bien que plutôt neutre sur le plan qualitatif, il est employé par J.-F. Copé de manière positive. Pour cela, il utilise une tournure un peu inhabituelle avec une juxtaposition (la virgule) plus une coordination (« et »), mettant sur le même plan les deux informations séparées par ce procédé. Avec «et » (et pas «mais »), on a la mise en évidence que les deux informations sont de même nature, à savoir positives. Son argument principal pour se légitimer de N. Sarkozy est donc cet engagement durant la campagne électorale, et il le tweete plusieurs fois, avec toujours ce goût pour l'intensité de l'engagement et l'assurance qu'il a été actif :

http://twitter.com/jf_cope/status/230634881812668417

Je me suis donné sans compter pour la campagne de Nicolas 
Sarkozy \#NiceMatinhttp://www.nicematin.com/societe/cope-a-lump-on-ne-doit-pas-avoir-detabou.949781.html ...

ou

http://twitter.com/jf_cope/status/227643548856639489

J'ai été très engagé aux côtés de Nicolas Sarkozy durant cette campagne \#RTLMatin

Les tweets sont le lieu de la construction d'un ethos discursif, ils contribuent à constituer pour les candidats une identité numérique, et visent à la faire percevoir dans les meilleures dispositions. Si pour F. Fillon et J.-F. Copé l'identité numérique coïncide avec leur identité hors-ligne, cette question dans le cadre du discours politique est néanmoins complexe, puisqu'une forme de délégation de l'écriture peut exister, une forme de "jeu de rôle » peut également se trouver plus spécifiquement sur le réseau ${ }^{5}$. D'ailleurs, la constitution de l'ethos peut aller jusqu'à la construction de toute pièce d'un ethos technodiscursif.

\subsection{L'éthos technodiscursif : la nouvelle grammaire du tweet du Parti Socialiste}

Dans certains cas, des personnalités politiques ont bien un compte Twitter, mais ne l'utilisent pas, ou très peu. C'est le cas pour certains cadres du PS, récents ministres en particulier. Leur identité numérique est alors prise en charge par le compte Twitter du parti, ce qui crée une forme d'hétérogénéité du dire. En linguistique, la théorie néo-dialogique de l'hétérogénéité discursive a notamment renouvelé la conception classique des différentes formes du discours (Authier-Revuz 1984). Pour mesurer ce qu'il en est de ce que nous appelons ethos technodiscursif, et prendre en compte la forme d'hétérogénéité qui le compose, prenons un exemple :

http://twitter.com/partisocialiste/status/243648347934695426

.@mlebranchu: «un vrai dialogue social, une vraie concertation, une vraie négociation»http://www.partisocialiste.fr/articles/marylise-lebranchu-un-vrai-dialogue-social-une-vraie-concertation-une-vraienegociation ...

Du point de vue de l'écriture du tweet, cet exemple indique l'appropriation d'un élément de la langue de Twitter, le @. En effet, ce signe permet normalement d'établir une mention, en créant un lien hypertextuel vers le compte d'un autre utilisateur. Par usage, c'est aussi devenu le moyen de citer un utilisateur, de lui répondre : le réseau s'est approprié le @ pour désigner bien souvent une identité virtuelle (le profil de quelqu'un, mais pas ce quelqu'un directement). Ce signe a une autre fonction en début de message : il permet de limiter aux followers (suiveurs) d'un compte l'envoi d'un tweet.

Les tweets de @partisocialiste (et des partis, ou des usagers, qui utilisent cette nouvelle méthode) combinent les deux fonctions du @ : ils le positionnent en début de tweet, ce qui focalise le message sur le profil désigné par ce @, mais en posant un point avant le @ (donc «.@ »), ils échappent à la limitation technologique d'envoi aux seuls followers (suiveurs), et restent dans le principe de mention. On a donc un nouveau moyen de citer le discours d'un autre, en mettant en scène un personnage à qui on donne la parole, comme dans un récit, dans lequel le narrateur donne la parole aux personnages :

http://fr.twitter.com/partisocialiste/status/244015195226267648

.@ManuelValls : «nous apportons une solution globale pour \#Marseille»http://www.partisocialiste.fr/articles/manuel-valls-nous-apportons-une-solution-globale-pour-marseille ...

Il s'agit d'un discours direct, le type de discours qui (selon la Grammaire Méthodique du français) serait la forme qui représenterait le plus fidèlement et le plus littéralement le

\footnotetext{
${ }^{5}$ Cette notion d'identité numérique est appuyée ici dans la mesure où c'est le discours politique qui nous intéresse : les choses sont très certainement différentes dans d'autres contextes d'usage de Twitter.
} 
distribués, au sens cognitif de ce terme, dans les environnements matériels de la production discursive (leur nature pratique voire technique, $[\ldots]$.

Ils sont également «temporellement antérieurs aux discours sur l'axe diachronique de leur production [...] mais sont localisés dans les relations entre le sujet et son environnement social, historique et technologique »(Paveau 2006 : 128). Les prédiscours ne sont pas des séquences discursives identifiables «mais des cadres préalables tacites, signalés dans les discours actuels par un certain nombre de phénomènes » (2011: en ligne). Dans la production de tweets politiques, et en particulier en conséquence de la condensation sémantique identifiée plus haut, de nombreux prédiscours sont identifiables.

Par exemple, J.-F. Copé souhaite réunir la droite décomplexée :

http://twitter.com/jf_cope/status/230640038436827136

Je défends moi-même une ligne, celle de la droite décomplexée. On ne doit pas avoir de tabou. \#NiceMatinhttp://www.nicematin.com/societe/cope-a-lump-on-ne-doit-pas-avoir-de-

tabou.949781.html ...

Et ne veut pas avoir de tabou:

http://twitter.com/jf_cope/status/230639365875974146

Je défends moi-même une ligne, celle de la droite décomplexée. On ne doit pas avoir de tabou. \#NiceMatinhttp://www.nicematin.com/societe/cope-a-lump-on-ne-doit-pas-avoir-detabou.949781.html ...

Il véhicule donc un prédiscours qui se formulerait par «la droite est complexée », ou au moins « il existe une certaine droite qui est complexée », discours qui s'intègre à « il y a un certains nombres de tabous dans la définition de la droite ». Son propre discours se fait donc en écho à ce prédiscours. Il élabore ensuite une sémantique spécifique de la droite décomplexée : pour cela, il a construit le sens d'une "droite complexée » qu'il identifie comme celle du «politiquement correct » (même s'il ne dit pas explicitement que c'est celle incarnée par son concurrent) :

http://twitter.com/jf_cope/status/239677107435876352

«La droite que j'aime, c'est aussi une droite totalement libérée du politiquement correct qui nous assomme » \#Chateaurenard

L'usage de la proposition relative, ici qui nous assomme, est une technique habituelle d'une certaine rhétorique politique : ce qui suit la relative est en effet une forme de préconstruit, et introduit un prédiscours formulable par « le politiquement correct nous assomme », présenté comme une évidence.

Par contraste, les prédiscours chez François Fillon sont d'une nature très différente, il s'inscrit dans une démarche désintéressée et noble de la politique. Cette conception de la Politique (avec un grand $\mathrm{P}$ ) accompagne une démarche globale :

http://twitter.com/FrancoisFillon/status/219189607932235777

Candidat à la fois pour s'opposer à une politique à contresens de l'Histoire et pour préparer la reconquête sans attendre \#Fillon\#UMP \#JDD

avec contresens de l'Histoire, il se pose comme celui qui incarne le "sens de l'Histoire ", donc comme l'autorité naturelle garante d'un certain cours des choses. Il est notable que F. Fillon se place en hauteur du débat, quitte à délaisser l'élection à la tête de l'UMP pour se positionner dans le contexte national plus large, en parlant de l'intérêt national :

http://es.twitter.com/FrancoisFillon/status/239301422557052928

L'intérêt national nous commande de repartir à la conquête des Français de nous rassembler de renouveler notre engagement politique \#NiceNS 
Ainsi, François Fillon véhicule des prédiscours sur des valeurs politiques ou historiques générales, avec lesquelles il se construit comme étant en adéquation : il existe un sens de l'Histoire, un intérêt national, et c'est implicitement lui qui est l'homme providentiel pour suivre ces voies: l'ethos discursif constitué se double d'un ethos qu'il affiche comme prétechnodiscursif, puisqu'il est tweeté comme mobilisant un savoir que l'on aurait sur l'homme.

\section{CONCLUSION}

Le tweet politique, loin d'être un simple relai supplémentaire de transmission de l'information, s'avère être une forme originale du discours politique, qui pourrait s'apparenter, s'il se stabilise davantage, à un genre de discours spécifique. En effet, de par ses contraintes matérielles et technologiques, il véhicule des formes qui peuvent devenir des «petites phrases» sur le plan discursif, et s'accompagner d'une intensité sémantique originale, par condensation et décontextualisation partielle, voire une recontextualisation par les moyens technologiques (que nous appelons techno-contextualisation). Ces caractéristiques s'accompagnent, pour l'utilisateur, de la constitution d'un ethos discursif, qui peut se doubler d'un ethos technodiscursif, grâce au maniement de certains codes propres au réseau. Plus encore, par l'insertion d'arrière-plans aux discours, un ethos prétechnodiscursif peut être véhiculé, dans la mesure où les tweets peuvent mobiliser des prédiscours qui développent, pour l'identité numérique mise en scène, un certain nombre d'éléments présentés comme étant préalables à l'énonciation. Le tweet politique est donc un lieu de renouvellement du discours politique, et un cadre intéressant pour observer certaines mutations des formes textuelles, sémantiques et discursives qui sont produites. Il permet également, si on s'y intéresse d'un point de vue linguistique, d'y voir une certaine forme de rhétorique, et de prendre cette techno-logie à la lettre, comme technè du logos. En effet, si comme le mentionne Breton (1998: en ligne), les sophistes «sont en réalité selon Aristote des technologues de la rhétorique, professeurs qui forment les logographes et leur apprennent à bien parler pour convaincre ", les tweets politiques s'inscrivent également dans cette forme de rhétorique. Alors que «le premier "geste» aristotélicien est celui qui enracine la rhétorique dans la sphère du pratique, en détachant ainsi la rhétorique de la sphère du technologique » (Parret 1988 : 79), le «geste » du tweet politique replonge la rhétorique politicienne dans la sphère du technologique, par le biais technolangagier. Certes, la dimension sophistique n'est pas absente des autres genres du discours politique, cependant le tweet politique, s'appuyant sur des propriétés technologiques, peut en accentuer les traits.

\section{REFERENCES}

AUTHIER-REVUZ J. (1984), « Hétérogénité(s) discursive(s) », Langages, n¹9, p. 98-111.

BENVENISTE E. (1966), Problèmes de linguistique générale T.1, Paris, Gallimard.

BRETON P. (1998), "L'argumentation dans la communication (N.T.I.C.) », in Usages et réception des médias - Journée d'études du 26 mai 1998, Ecole Normale Supérieure de Fontenay/Saint-Cloud, http://www.hatt.nom.fr/rhetorique/artic110.htm

CHAUVIN-VILENO A. (2002), «Ethos et texte littéraire. Vers une problématique de la voix », Semen [En ligne], 14/2002, mis en ligne le 30 avril 2007, consulté le 12 janvier 2012. URL : http://semen.revues.org/2509

KRIEG-PLANQUE A., Ollivier-Yaniv C. (2011), « Poser les 'petites phrases' comme objet d'étude », Communication \& Langages, Paris, Editions Necplus, n¹68, juin 2011, p.17-22.

LONGHI J., SARFATI, G.-E. (2012), Dictionnaire de pragmatique, Paris, Armand Colin. 
MAINGUENEAU D. (1999), «Ethos, scénographie, incorporation » in R. Amossy (dir.), Images de soi dans le discours. La construction de l'ethos, Lausanne-Paris, Delachaux Niestlé, 1999, p. 75-100.

PARRET H. (1988), « Au-delà de la rhétorique du juridique : justifier par l'éthique, légitimer par l'esthétique », Droit et société, n 8 , p. 77-89.

PAVEAU M.-A. (2006), Les prédiscours. Sens, mémoire, cognition, Paris, Presses Sorbonne nouvelle.

PAVEAU M.-A. (2011), «Quelles données entre l'esprit et le discours ? Du préconstruit au prédiscours ", in A. Azouzi (éd), L'analyse du discours. Notions et problèmes, Tunis, Sahar Éditions, http://hal.archives-ouvertes.fr/docs/00/66/00/71/PDF/paveau-ad-10.12.09.pdf

PAVEAU M.-A. (2012), "Activités langagières et technologie discursive. L'exemple de Twitter », La pensée du discours [carnet de recherche]

RIEGEL M., PELLAT J.C., RIOUL R. (2004), Grammaire méthodique de français, Paris, PUF.

TLFI : Trésor de la langue française informatisé.

Corpus des tweets analysés : http://corpusdetweetspolitiques.blogspot.fr/

Julien LONGHI

Université de Cergy-Pontoise 